Onkologe 2011 · 17:194-196

DOI 10.1007/s00761-010-1997-1

(c) Springer-Verlag 2011

\author{
L. Weißbach ${ }^{1} \cdot$ K. Höffken ${ }^{2}$ \\ ${ }^{1}$ Stiftung Männergesundheit, Berlin \\ 2 Universitätsklinikum Jena
}

\title{
Der tägliche Spagat zwischen Ethik und Ökonomie
}

"Der ärztliche Beruf ist kein Gewerbe“ das ist der erste Satz der Musterberufsordnung, klar und schnörkellos. Da wird ein großes Wort gelassen ausgesprochen (wie es Goethe seinem Thoas in den Mund legt). Der Satz zeugt von Selbstbewusstsein und einem klaren Verständnis des ärztlichen Auftrags. Der Unterschied zwischen einem Gewerbe und einem freien Beruf füllt mehrere laufende Meter juristischer Literatur - und läuft doch auf auf ein einziges Wort hinaus: Profit. Ein Gewerbe wirtschaftet profitorientiert, während der freie Beruf aufwandsorientiert, d. h. bei einer angemessen honorierten Leistung nicht dem Gewinn verpflichtet sein sollte.

In der Tat lässt sich Gewinnstreben mit dem ärztlichen Ethos, wie es seit Jahrtausenden tradiert wurde, schwer vereinbaren. Auch wenn kein Arzt - wie ein Bundeswehrrekrut - auf Hippokrates vereidigt wird: Das von ihm überlieferte Prinzip „Primum nihil nocere“ gilt heute wie früher. Damit werden der Patient und sein Wohl ins Zentrum ärztlichen Handelns gestellt. Dies deckt sich mit der ursprünglichen Motivation vieler Ärzte, denn die meisten haben ihren Beruf ergriffen, weil sie helfen und Leid lindern wollten. Die Realität jedoch sieht anders aus. Die solidarisch finanzierte Gesundheitsversorgung stößt an ihre Grenzen, wenn medizinischer Fortschritt teurer, die Einnahmen aus der Erwerbsarbeit rückläufig werden und das Gesundheitssystem dazu beiträgt, sich die Klientel selbst zu schaffen (Beitrag Früherkennung). Geld wird knapp und der Mangel droht verwaltet zu werden. $\mathrm{Ob}$ in dieser Situation eine Beschränkung von solidarisch finanzierten Leistungen durch den Staat mit dem Grundgesetz vereinbar ist, fragt Huster in seinem Beitrag.

\section{( Gewinnstreben lässt sich mit dem ärztlichen Ethos schwer vereinbaren}

In diesem Kontext wird zunehmend (auch laut) darüber nachgedacht, inwieweit es statt eines Anrechts auf Gesundheit (s. Beitrag Lang) eine Gesundheitspflicht für das einzelne Individuum gibt. Auch wenn dieses Thema $z$. T. noch Inhalt von Science-Fiction-Romanen ist, geben die Möglichkeiten der modernen Gendiagnostik zu Spekulationen Anlass: Feststellbare Prädispositionen für vorhersagbare Erkrankungen könnten dem Einzelnen die Verpflichtung auferlegen, seinen Lebensstil daran auszurichten, um so eventuelle Manifestationen der Erkrankung zu verhindern. Andernfalls bestünde keine Leistungspflicht der Kostenträger.

Gleichzeitig sollen die Leistungserbringer verstärkt in den Wettbewerb eintreten, unter anderem, weil sich die politischen Gestalter hiervon Spareffekte erhoffen. Versorgungseinrichtungen werden mehr und mehr als Betriebe im wirtschaftlichen Sinne verstanden und entsprechend geführt. Doch das Ideal des altruistischen Arztes lebt in der Gesellschaft fort - allen Debatten um Rationierung, Kopfpauschalen oder Abrechnungsbetrug zum Trotz: Noch immer genießen Ärzte das mit Abstand höchste Ansehen in der Gesellschaft, wie die Berufsprestigeskala von Allensbach mit schöner Regelmäßigkeit zeigt. Im medizinischen Alltag hingegen zwingt die zunehmende Kluft zwischen ethischem Anspruch und betriebswirtschaftlicher Realität viele Ärzte in einen Spagat. Bartsch und Maio beleuchten, wie ökonomische Zwänge ärztliches Handeln im Alltag beeinflussen: Was sich nicht rechnet, findet nicht statt. Und das, obwohl angeblich der Patient im Mittelpunkt steht.

Kliniken in privater Trägerschaft bringen zweistellige Renditen und werden als leuchtendes Beispiel für kommunale Träger zitiert. Ethos und Zuwendung bleiben da auf der Strecke. Doch was die einen Ökonomie nennen, ist für andere Kommerzialisierung oder Vergewerblichung.

Es wäre zu einfach, die Schuld an diesen Zuständen „der Politik“ oder „den Ökonomen“ zuzuweisen, die heute fast alle Schlüsselpositionen im Gesundheitswesen besetzen. Ärzte tragen Verantwortung: zunächst ganz konkret für jeden ihnen anvertrauten Patienten. Die Indikation wird zunehmend zu einer ökonomischen Zwangsentscheidung, der ein Patient ausgeliefert ist. Die Handlung verantwortet aber in letzter Konsequenz der behandelnde Arzt. Medizinische Kompetenz verpflichtet jedoch zu mehr. Vier am Patienten handelnde Ärzte haben die Frage beantwortet, was sie sich wünschen, um ethisch angemessen handeln können. Eine wichtige Antwort war: ein System, in dem die Ärzte sich einmischen. Letztlich muss aber die Frage erlaubt sein, was diese mutige Einmischung bewirken kann, wenn das zur Kostenbewertung und 
Hier steht eine Anzeige.

黛 Springer 
-übernahme installierte Gremium zweifelhaft legitimiert ist und keine entscheidende Mitwirkung der Patienten, die angeblich im Mittelpunkt stehen, ermöglicht (s. Beitrag Lang).

Hoffentlich ermutigen die Beiträge dieses Hefts trotzdem den einen oder anderen Leser dazu, sich zukünftig ebenfalls einzumischen - zum Wohle des Patienten und nicht, um wirtschaftliche Zielvorgaben oder Mindestmengen zu erreichen.

Dann wäre der täglich zu beobachtende Spagat nicht einer zwischen Skylla und Charybdis.

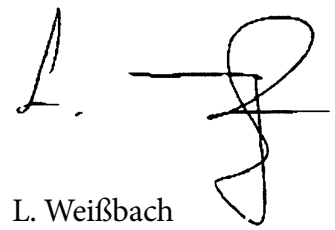

Für die Herausgeber des Schwerpunkthefts

\section{Horran}

K. Höffken

Für die Herausgeber

\section{Korrespondenzadresse \\ Prof. Dr. L. Weißbach}

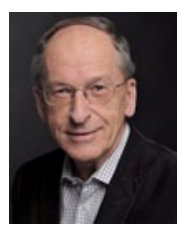

Stiftung Männergesundheit

Reinhardtstraße 2, 10117 Berlin

weissbach@stiftung-

maennergesundheit.de

\section{Stipendien der José Carreras Leukämie-Stiftung}

Vier Stipendien

für Nachwuchswissenschaftler

Als gemeinnütziger Verein leistet die José Carreras Leukämie Stiftung einen wesentlichen Beitrag zur Bekämpfung von Leukämien und verwandten Blutkrankheiten. Dazu werden mit Förderschwerpunkt experimenteller und klinischer Forschung vier Stipendien für Nachwuchswissenschaftler ausgeschrieben.

\section{Die Stipendien sind für Mediziner und} Naturwissenschafter bestimmt, die bereits Erfahrung in der hämatologischen, zellbiologischen, immunologischen oder psychoonkologischen Forschungsarbeit besitzen, an einer wissenschaftlichen Institution in Deutschland arbeiten und nicht älter als 35 Jahre sind. Für das Stipendium ist ein Förderbetrag von 41.400 Euro pro Jahr vorgesehen. Zuwendungen werden zunächst für ein Jahr bewilligt und können auf Antrag um höchstens zwei Jahre verlängert werden.

Anträge können vom Antragsteller persönlich oder von der Forschungsinstitution gestellt werden. Antragsschluss ist der 31.03.2011.

Ausschreibungsbedingungen und Antragsunterlagen sind im Internet abzurufen unter www.carreras-stiftung.de oder können bei der José Carreras Leukämie-Stiftung, Elisabethstr. 23, 80798 München, angefordert werden.

Quelle: www.carreras-stiftung.de

\section{DKG-Internetportal} AGO Meeting 2011: Experten diskutieren State of the Art beim Mammakarzinom

Die Kommission „Mamma“ der Arbeitsgemeinschaft Gynäkologische Onkologie e.V. (AGO) hat die aktuelle Version ihrer „Empfehlungen zur Therapie primärer und fortgeschrittener Mammakarzinome", die jährlich revidiert werden, im Rahmen des „AGO Mammakarzinom Update 2011" in Frankfurt vorgestellt. Das DKG-Internetportal berichtet über die Veranstaltung, um Ärzten einen zeitnahen und kompakten Überblick über die neuesten Entwicklungen zu geben. So findet sich auf der Website ein interdisziplinäres Expertengespräch unter dem Vorsitz von Prof. Dr. Anton Scharl, Leiter des Brustzentrums St. Marien Amberg, zur adjuvanten Therapie beim Mammakarzinom. Schwerpunkte sind unter anderem die Bedeutung von Bisphosphonaten als adjuvante Therapiestrategie bei definierten Patientengruppen, die Neubewertung der Therapieempfehlungen bei triple-negativen oder mutationsbedingten Mammakarzinomen und die Neubewertung der Indikation zur Strahlentherapie bei älteren Patientinnen. Zu weiteren Angeboten gehören ein Interview mit Prof. Scharl, das einen kompakten Überblick über das AGO-Meeting gibt, sowie ein Interview mit dem Leiter der GBG Forschungs $\mathrm{GmbH}$, Prof. Dr. Gunter von Minckwitz, Neu-Isenburg, über zehn Jahre German Breast Group (GBG).

Quelle: Deutsche Krebsgesellschaft, www.krebsgesellschaft.de 